\title{
One-Way Spinning Top
}

\author{
Kenneth Brecher designs spinning tops using mathematical constants, \\ including the Feigenbaum constant, which produces a top with a \\ preferential spinning direction.
}

By Katherine Wright

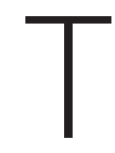

he spinning top is a classic childhood toy, with its twirls and wobbles providing hours of entertainment. But for Kenneth Brecher, tops are more than toys: They are a way to demonstrate physics and math in action. For the last six years, the retired astrophysicist from Boston University has been optimizing the design of spinning tops so that they pirouette for longer, something he does by incorporating mathematical constants into their dimensions. Brecher presented his latest top-a one-way spinning device called a rattleback top-at the recent 2021 Bridges conference, a meeting on math-inspired art (see Physics Abounds at Conference on Math and Art).

Brecher's interest in spinning tops stems from his work on understanding the spinning motion of neutron stars-he was

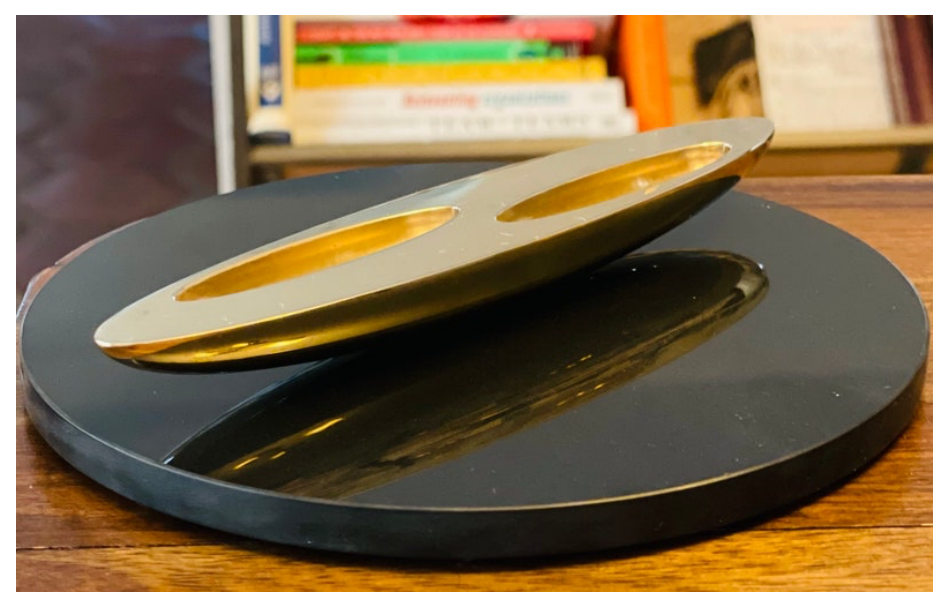

When spun clockwise, this spinning top designed by Kenneth Brecher twirls briefly before wobbling and then reversing its spinning direction.

Credit: Karen Brecher one of the first to show that they wobble, or precess. Brecher has long used tops in classroom demonstrations, but it wasn't until he was rendered immobile by knee surgery in 2015 that he decided to start making his own. "While I was recovering, my daughter Karen suggested I do something with all my classroom demonstration stuff," he says. They started a small funding drive through the crowdfunding platform Kickstarter and within three days had enough seed money to start a top-making enterprise called Sirius Enigmas.

That Kickstarter drive was for Brecher's PhiTOP, an egg-shaped, metallic top that when spun from a horizontal position will suddenly stand erect and start rotating on one of its narrow ends. While still not fully understood, the transition comes from friction between the top and the underlying platform that exerts an upward force on the top's center of mass. The phenomenon was first investigated in the 19th century by Lord Kelvin. "There is a long tradition of physicists fooling around with tops," Brecher says.

Brecher's version of the top has a height-to-width ratio of 1.618 (called the golden ratio, or phi) and was inspired by a classroom demonstration he did with real eggs. "If you spin a hardboiled egg, it will stand up; if it's raw, it doesn't," he says. Playing around with making replica eggs, Brecher discovered that the longest spin times-about 6 minutes-occurred for eggs having a height-to-width ratio of about 1.6. But to make it more fun he decided to go with phi.

Brecher has since made tops that incorporate other constants, including the coin-shaped PiTOP, which has a radius-to-thickness ratio of $\pi$, and a pebble-shaped eTOP, which has a radius-to-thickness ratio of $e / 2$. These tops are all designed to spin for as long as possible and can spin in both 


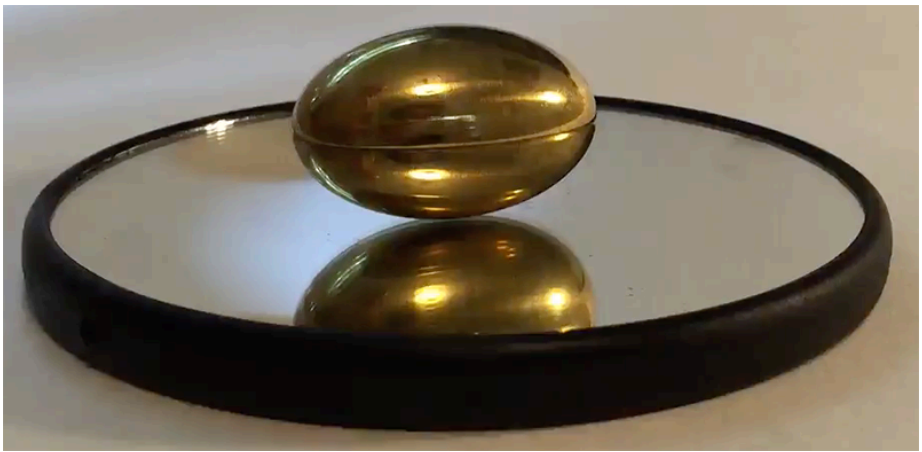

The PhiTOP was the first spinning top that Brecher made incorporating physics and math constants.

Credit: Kenneth Brecher

clockwise and counterclockwise directions. But his latest creation, the DeltaCELT, a canoe-shaped rattleback top with two ellipsoidal pits carved into its surface, is different: It likes to spin only one way.

This particular rattleback was designed so that the ratio of its major axis to its minor axis equals the Feigenbaum constant $\delta$, which has a value of around 4.669. The constant determines the rate at which chaos develops in chaotic systems, such as turbulent flows. Initially, Brecher wanted to make a chaotic top, but that turned out to be difficult, so he turned to the next best thing-a top with some quirky dynamics.

If spun counterclockwise, the DeltaCELT behaves like any other top, spinning freely until it eventually slows because of friction. But if spun clockwise, something odd happens: the top twirls briefly, slows down, stops, and then reverses its spinning direction. Brecher says that this behavior comes from the asymmetric shape of the top, which induces a torque that opposes clockwise rotation and converts rocking motion into counterclockwise rotation.

Brecher says he was inspired to make this top using the Feigenbaum constant after his long-time friend, the physicist
Mitchell Feigenbaum, died in 2019. The two had been friends for 50 years, meeting first when they were in graduate school at the Massachusetts Institute of Technology. "I was coming back from speaking at his memorial two years ago, and I thought, I've got to do something with the Feigenbaum constant," he says. "A

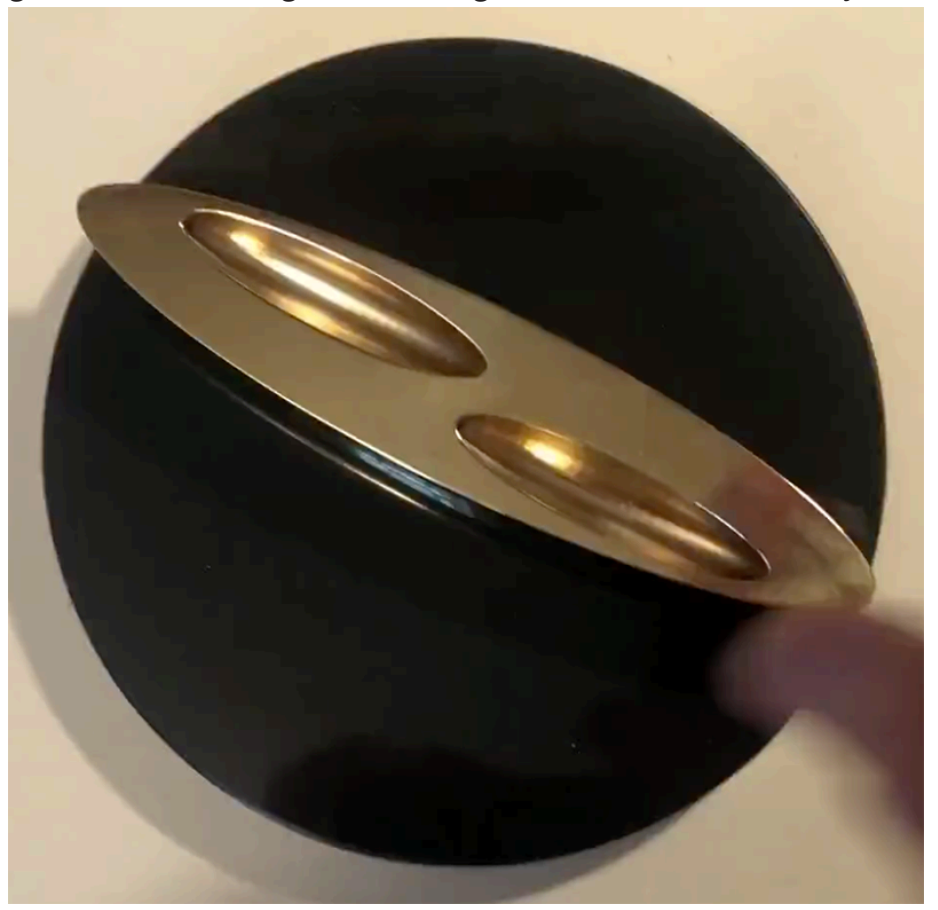

Unlike other spinning tops, a rattleback has a preferential spinning direction, which comes from its asymmetric shape. When spun clockwise, the top rapidly slows down to a stop and begins to rock (or "rattle") back and forth. It then transforms this rocking motion into rotation in a counterclockwise direction.

\section{Credit: Kenneth Brecher}

top seemed like the right thing."

Katherine Wright is the Deputy Editor of Physics. 